\title{
External Auditory Canal Hemangioma: Case Report
}

\author{
D. J. Verret, M.D., ${ }^{1}$ C. Spencer Cochran, M.D., ${ }^{1}$ Robert J. DeFatta, M.D., Ph.D., ${ }^{1}$ \\ and Ravi N. Samy, M.D., F.A.C.S. ${ }^{1}$
}

A patient with a hemangioma completely within the external auditory canal is reported.

KEYWORDS: External auditory canal, hemangioma, hearing loss

\section{CASE REPORT}

A 31-year-old Hispanic man presented with complaints of hearing loss and the sensation of a foreign body in his right ear. He had no symptoms of dizziness, otorrhea, pain, or tinnitus. On otoscopic examination, a mass was seen filling the right external auditory canal (EAC). Its medial extent could not be visualized (Fig. 1). The mass did not extend beyond the meatus into the concha; the auricle was normal. An audiogram showed a mild conductive hearing loss of the right ear. A CT scan of the temporal bones without contrast showed a mass in the EAC measuring about $16 \mathrm{~mm}$. Bony erosion of the EAC was limited, and there appeared to be no involvement of the tympanic membrane (Fig. 2). There was no involvement of the temporal lobe or internal carotid artery by the tumor. A biopsy of the lesion was performed.

\footnotetext{
${ }^{1}$ Department of Otolaryngology-Head and Neck Surgery, University of Texas Southwestern Medical Center, Dallas, Texas.

Address for correspondence and reprint requests: D. J. Verret, M.D., Department of Otolaryngology-Head and Neck Surgery, University of Texas Southwestern Medical Center, 5323 Harry Hines Blvd., Dallas, TX 75390-9035 (e-mail: Daniel.Verret@parknet. pmh.org).
}

The patient was given options of observation or surgical resection. He chose to undergo surgery. Complete surgical resection was performed via a postauricular approach. As noted on preoperative imaging, the tumor did not involve the tympanic membrane. The skin of the EAC was reconstructed with the use of a split-thickness skin graft measuring 0.008 inch thick and obtained from the right lower extremity. The patient healed well after surgery with no evidence of tumor recurrence.

\section{DIsCusSION}

The patient was diagnosed with a hemangioma of the EAC. There have been few reports in the English literature of a hemangioma residing entirely

Skull Base 2007;17:141-144. Copyright (C) 2007 by Thieme Medical Publishers, Inc., 333 Seventh Avenue, New York, NY 10001, USA. Tel: +1(212) 584-4662. 2006.

Accepted: February 16, 2006. Published online: December 20, DOI 10.1055/s-2006-953512. ISSN 1531-5010. 


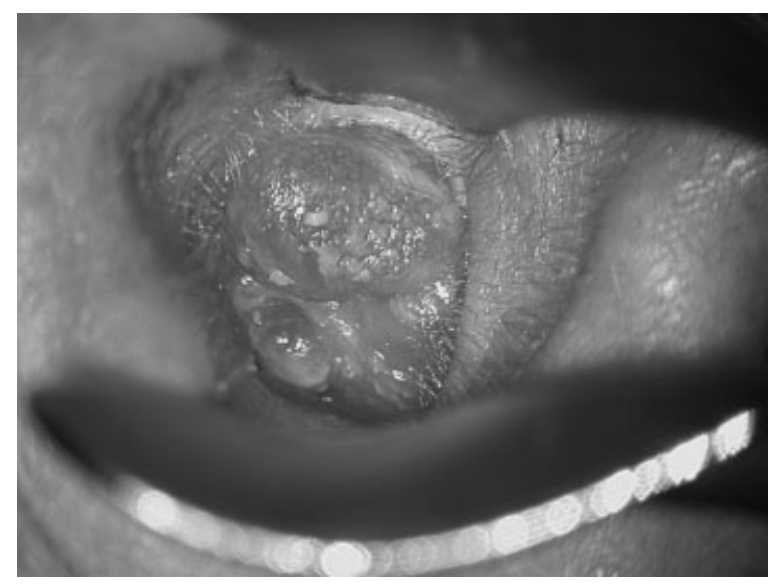

Figure 1 Image of a mass filling the external auditory canal.

within the EAC. Most hemangiomas of the EAC are extensions from auricular skin. The first report of a hemangioma within the EAC was by Freedman and colleagues in $1972 .{ }^{1}$ They described two men in

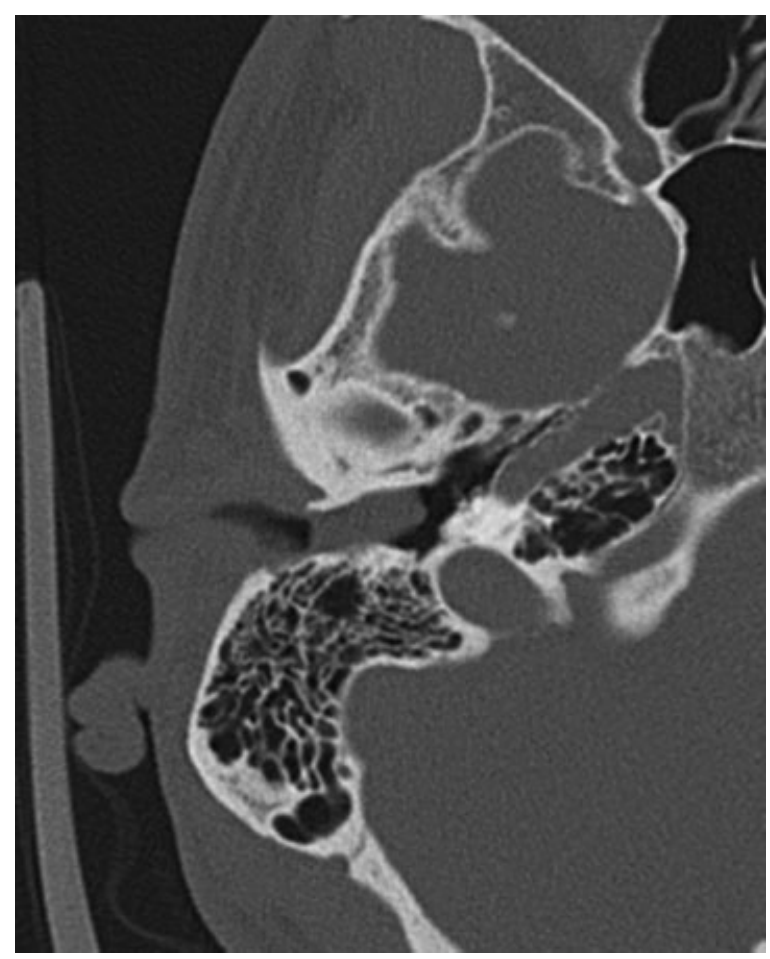

Figure 2 Axial bone CT scan showing a soft tissue mass in the EAC associated with limited bony erosion without involvement of the tympanic membrane. EAC, external auditory canal. their sixth decade with vascular lesions of the EAC that extended onto the tympanic membrane. In the first patient, excision of the lesion included the posterior canal skin and half of the tympanic membrane via an endaural approach. At last follow-up (5 years), the patient showed no recurrence of the tumor. He had no change in hearing after surgery or at last follow-up. The second patient underwent transcanalicular excision of the lesion, which included resection of the tympanic membrane. At his 18-month follow-up, the patient showed no evidence of recurrence and his hearing was unchanged from baseline.

In 1983, Kemink and associates ${ }^{2}$ reported on a 52-year-old man with a hemangioma who underwent tympanoplasty with mastoidectomy to excise the lesion. The patient initially underwent excision of the lesion on the posterior canal wall via a postauricular approach. However, the bone deep to the lesion appeared soft and bled easily. Although the mastoid cavity was entered, no tumor was identified there.

In 1990, Jackson and colleagues ${ }^{3}$ reported the first EAC hemangioma in a woman. Their patient, 60 years old, presented with an EAC lesion initially misdiagnosed as otitis externa. Excisional biopsy revealed findings consistent with hemangioma. Two months after her initial diagnosis, the patient had a recurrent mass filling the EAC and conductive hearing loss. She underwent temporal bone resection. At surgery, the tumor was seen to involve the posterior wall of the EAC and tympanic membrane but not the middle ear or ossicles. The patient underwent meatoplasty with tympanoplasty. ${ }^{3}$

These initial reports of hemangiomas in the EAC included involvement of the tympanic membrane. In 1987, Hawke and van Nostrand ${ }^{4}$ published a report of a hemangioma contained wholly within the EAC with no involvement of the tympanic membrane in a 55-year-old man with a 4-month history of blood-tinged discharge from the right ear. Under direct microscopy, a friable "polyp" was seen in the patient's right EAC. Due to manipulation, significant arterial bleeding occurred. 
The bleeding did not respond to adrenalin-soaked gauze and required electrocauterization under general anesthesia. Although our patient underwent biopsy before surgical resection without complications, one must be prepared to deal with significant bleeding. Hawke and van Nostrand ${ }^{4}$ reported no further excision beyond the patient's office biopsy. Two weeks postoperatively, the patient had no sign of recurrence.

In the previously reported cases, most EAC hemangiomas also involved the tympanic membrane. In our case, as in that of Hawke and van Nostrand, ${ }^{4}$ the tympanic membrane was not involved. Furthermore, neither the middle ear nor the ossicular chain was involved in any of the cases. As in our case, the bony auditory canal appeared to be involved only superficially without mastoid involvement.

The pathologic description of these tumors typically shows a surface of keratinized, stratified, squamous epithelium. Beneath this level is dense fibrous connective tissue with numerous large, thinwalled, vascular spaces. ${ }^{3}$ In the report by Hawke and van Nostrand, ${ }^{4}$ the endothelial lining stained positively for both antifactor-VIII antibody and Ulex europeus I agglutinin, which provided additional evidence that the vascular channels were lined with vascular epithelium.

\section{SUMMARY}

Although hemangiomas arising solely in the EAC are rare entities, they should be considered in the differential diagnosis of vascular-appearing masses in the EAC. A biopsy can be performed in clinic but is not prudent because significant bleeding can occur. CT with contrast or MRI with gadolinium may better delineate the vascular nature of the mass without the risk of biopsy complications. Every case thus far reported has been easily treated with complete surgical excision with no reported morbidities. That these lesions do not involve the middle ear means that complete excision, while being curative, can also be accomplished with minimal risk to hearing.

\section{REFERENCES}

1. Freedman SI, Barton S, Goodhill V. Cavernous angiomas of the tympanic membrane. Arch Otolaryngol 1972;96: 158-160

2. Kemink JL, Graham MD, McClatchey KD. Hemangioma of the external auditory canal. Am J Otol 1983;5:125-126

3. Jackson CG, Levine SC, McKennan KX. Recurrent hemangioma of the external auditory canal. Am J Otol 1990;11:117-118

4. Hawke M, van Nostrand P. Cavernous hemangioma of the external ear canal. J Otolaryngol 1987;16:40-42 